\title{
Implicit Learning and Dyslexia
}

\author{
Vasiliki Folia, ${ }^{a, b, c}$ Julia Uddén, ${ }^{a, b, c}$ Christian Forkstam, ${ }^{a, b, c}$ \\ Martin Ingvar, ${ }^{b, d}$ Peter Hagoort, ${ }^{a, c}$ \\ and Karl Magnus Petersson ${ }^{a, b, c, d}$
}

\author{
a Max Planck Institute for Psycholinguistics, Nijmegen, the Netherlands \\ ${ }^{b}$ Cognitive Neurophysiology Research Group, Stockholm Brain Institute, \\ Karolinska Institute, Stockholm, Sweden \\ ${ }^{c}$ F. C. Donders Centre for Cognitive Neuroimaging, Radboud University, \\ Nijmegen, the Netherlands \\ ${ }^{d}$ Cognitive Neuroscience Research Group, Universidade do Algarve, Faro, Portugal
}

\begin{abstract}
Several studies have reported an association between dyslexia and implicit learning deficits. It has been suggested that the weakness in implicit learning observed in dyslexic individuals may be related to sequential processing and implicit sequence learning. In the present article, we review the current literature on implicit learning and dyslexia. We describe a novel, forced-choice structural "mere exposure" artificial grammar learning paradigm and characterize this paradigm in normal readers in relation to the standard grammaticality classification paradigm. We argue that preference classification is a more optimal measure of the outcome of implicit acquisition since in the preference version participants are kept completely unaware of the underlying generative mechanism, while in the grammaticality version, the subjects have, at least in principle, been informed about the existence of an underlying complex set of rules at the point of classification (but not during acquisition). On the basis of the "mere exposure effect," we tested the prediction that the development of preference will correlate with the grammaticality status of the classification items. In addition, we examined the effects of grammaticality (grammatical/nongrammatical) and associative chunk strength (ACS; high/low) on the classification tasks (preference/grammaticality). Using a balanced ACS design in which the factors of grammaticality (grammatical/nongrammatical) and ACS (high/low) were independently controlled in a $2 \times 2$ factorial design, we confirmed our predictions. We discuss the suitability of this task for further investigation of the implicit learning characteristics in dyslexia.
\end{abstract}

Key words: artificial grammar learning; structural mere-exposure effect; dyslexia; inferior frontal cortex; basal ganglia

\section{Introduction}

During the acquisition of reading and writing skills, children develop the ability to represent aspects of the phonological component of language by an orthographic rep-

Address for correspondence: Karl Magnus Petersson, Max Planck Institute for Psycholinguistics, P.O. Box 310, 6500 AH Nijmegen, the Netherlands. Voice: +0031-2436-10984; fax: +0031-2436-10652. karl.magnus.petersson@fcdonders.ru.nl resentation and relate this to a visuographic input-output code. This is typically achieved by means of a supervised learning process (i.e., teaching), in contrast to natural language acquisition, which is largely a spontaneous, non-supervised, and self-organized acquisition process (Petersson, 2005a; Petersson, Ingvar, \& Reis, 2009). Aspects of language can also be an object of metalinguistic awareness: the intentional and explicit control over aspects of phonology, syntax, semantics, 
and discourse, as well as pragmatics. Children gradually create explicit representations and acquire processing mechanisms that allow for reflecting and analyzing different aspects of language use (Karmiloff-Smith, Grant, Sims, Jones, \& Cuckle, 1996). When children subsequently learn to read, this has repercussions on the phonological representations of spoken language (Morais, 1993; Petersson, Reis, Askelöf, Castro-Caldas, \& Ingvar, 2000; Petersson, Reis, \& Ingvar, 2001; Ziegler \& Goswami, 2005; see Goswami, this volume). Learning to read involves both explicit as well as implicit processes; typically children initially learn grapheme-phoneme mappings explicitly, after which they apply and continue to learn how phonology is mapped onto its written representation implicitly (Gombert, 2003; Petersson \& Reis, 2006; Ziegler \& Goswami, 2005). Karmiloff-Smith (1992) proposed that cognitive development relies on implicit/procedural learning mechanisms to initiate the setup of a new stage of representational development. A deficit in implicit acquisition mechanisms might therefore have a negative impact on the acquisition of reading and writing skills and therefore affect literacy acquisition. Dyslexia is rarely studied within the framework of learning, and a deficit in implicit learning might contribute to difficulties associated with dyslexia (Howard, Howard, Japikse, \& Eden, 2006; see also Bennet et al., this volume; Menghini et al., this volume; Stoodley et al., this volume). Recently, Howard et al. (2006) provided evidence suggesting that the weakness in implicit learning observed in dyslexic individuals might be narrowed down to paradigms that involve sequential processing and they argued that the implicit sequence learning deficit in dyslexia is associated with selective deficits in the frontostriatal-cerebellar circuits that underlie implicit sequence learning. It has been shown that fronto-striatal circuits are involved in sequence processing after implicit grammar acquisition (Forkstam, Hagoort, Fernandez, Ingvar, \& Petersson, 2006; Petersson, Forkstam, \& Ingvar, 2004).

\section{Implicit Learning}

Humans are equipped with acquisition mechanisms that extract structural regularities implicitly from experience without the induction of an explicit model (Reber, 1967, 1993; Stadler \& Frensch, 1998). This capacity was explored in the seminal work of Reber (1967), showing that humans can successfully classify strings generated from an implicitly acquired artificial grammar and proposed that this process is intrinsic to natural language acquisition. Following this suggestion, it has been argued that artificial grammar learning (AGL) is a relevant model for investigating aspects of language learning in infants (Gomez \& Gerken, 2000), exploring differences between human and animal learning relevant to the narrow faculty of language (Hauser, Chomsky, \& Fitch, 2002), and language learning in adults (Friederici, Steinhauer, \& Pfeifer, 2002; Petersson et al., 2004). We suggest that it can serve as a device for investigating the implicit aspects of structure learning related to reading and writing acquisition as well. Reber (1967) suggested that humans can acquire implicit knowledge of the underlying structure of grammar through a statistical learning process and that the acquired knowledge is put to use during grammaticality classification. Reber (1967; but see Reber, 1993) argued that implicit learning mechanisms abstracted "rule-based" knowledge, and more recent studies seem to suggest that dual mechanisms might be engaged (Forkstam et al., 2006; Knowlton \& Squire, 1996; Meulemans \& Van der Linden, 1997). Following Reber (1967) and Seger (1994), Forkstam \& Petersson (2005) adapted four proposed defining characteristics of implicit learning: (a) explicit access is limited to the knowledge acquired - subjects typically cannot provide a sufficient explicit account of what they have learned; (b) the nature of the knowledge acquired is more complex than simple associations or simple exemplarspecific frequency counts; (c) implicit learning does not involve explicit hypothesis testing, but is an automatic (incidental) consequence of 
the type and amount of processing performed on the stimuli; and (d) implicit learning does not rely on declarative memory mechanisms that engage the medial temporal lobe memory system.

\section{Dyslexia: An Implicit Learning Deficit?}

Developmental dyslexia is commonly defined as a reading disability, a deficit in learning to spell and write, occurring in children despite normal intelligence, no sensory or neurological impairment, and conventional instruction and socioeconomic opportunity (Dilling, Mombour, \& Schmidt, 1991; Habib, 2000; Shaywitz, 1998). However, dyslexia is rarely studied in the framework of the contemporary learning literature (Howard et al., 2006). Learning to read involves both explicit as well as implicit processes; children initially learn the grapheme-phoneme correspondence explicitly, typically in a supervised manner, after which they apply and continue to learn them implicitly in an unsupervised manner (Gombert, 2003). A deficit in implicit learning might contribute to difficulties associated with dyslexia, but the literature on implicit learning and dyslexia has yielded mixed results (Howard et al., 2006). Most studies of implicit learning in dyslexics have investigated serial reaction time (SRT) types of tasks and there are, to our knowledge, only two studies that investigate artificial grammar learning (AGL). For a recent review of these experimental task, see Forkstam and Petersson (2005).

An important weakness of all studies of implicit learning in dyslexics to date is that they lack a developmental design (Goswami, 2003). Another weakness of some of the studies is that they report null findings. These null findings are difficult to interpret in the context of small study samples and experimental designs that are not always carefully controlled. Therefore, it is likely that the absence of significant results reflects a lack of statistical power as well as the presence of confounding factors. On the other hand, the conflicting literature on im- plicit learning and dyslexia might suggest that it is not enough to investigate simple implicit acquisition tasks or just to contrast implicit and explicit learning. In this brief but comprehensive review, we will give priority to those studies which find an implicit learning deficit in dyslexics, but we will also comment on those which report null findings, beginning with those studies that have investigated SRT-type tests and subsequently turning our attention to the AGL studies.

Vicari et al. (2003) reported deficient implicit learning in dyslexic children on visuomotor SRT-type tasks that used sequences of colors. They also included a test of declarative (explicit) memory capacities. Their main finding suggests that individuals with developmental dyslexia are impaired in the acquisition of implicit sequence knowledge, while there was no significant difference between the dyslexic and control groups in terms of explicit sequence learning. Some studies have reported null-findings on similar SRT-type tasks (Kelly, Griffiths, \& Frith, 2002; Waber et al., 2003), and Rüsseler et al. (2006) questioned the implicit learning deficit in dyslexia based on these and their own null findings. However, Waber et al. (2003) investigated a sample of children with "heterogeneous learning problems," which makes their findings difficult to interpret in the context of dyslexia, and although there was no significant learning difference between the dyslexic and normal readers in Rüsseler et al. (2006), the dyslexic subjects showed consistently longer response times (RTs) on the SRT task compared to the normal controls. This was also the case in Kelly et al. (2002). Of inportance, in a follow-up study, Vicari et al. (2005) used the classical SRT task as well as an implicit mirror drawing test, and showed that the children with developmental dyslexia were impaired on both tasks. Their SRT results suggest a deficit in sequential learning and that the deficit does not depend on the material being learned (with or without motor sequence of response action), but only on the implicit character of the task. These behavioral 
findings were further replicated in an fMRI study of adult dyslexics (Menghini, Hagberg, Caltagirone, Petrosini, \& Vicari, 2006). Consistent with this perspective, both Stoodley et al. (2006) and Howard et al. (2006) provided further evidence that the implicit learning deficits observed in dyslexic individuals can be narrowed down to paradigms that involve sequential processing. Stoodley et al. (2006) found significant differences in implicit learning between good and poor readers on the SRT task. In addition, the dyslexic group showed less of an RT decrease on the repeated sequence, while the RTs were similar to that of the control group on the random trials. Recently, Sperling et al. (2004) argued that poor implicit learning could hinder the establishment of good phonological processing as well as learning orthographic-phonological representations, whereas Gombert (2003) proposed that children with dyslexia have a phonological deficit that prevents normal implicit learning of linguistic regularities and hence interferes with reading development. Howard et al. (2006) showed that adult dyslexics are impaired on implicit acquisition in an alternating (higherorder) SRT task in which sequential dependences exist across nonadjacent elements. They compared the performance on the alternating SRT task with the performance on a simple spatial context learning task in which the global configuration of a display cues the location of a search target. Their results suggest that college students with a history of dyslexia are impaired in implicit higher-order sequence learning, but unimpaired in spatial context learning. They also argue that evidence from functional neuroimaging, and transcranial magnetic stimulation investigations in patients suggest that sequence learning depends on fronto-striatalcerebellar circuitry and that the acquisition of nonadjacent, higher-order, sequential regularities calls on fronto-striatal-cerebellar circuitry, whereas spatial contextual learning depends on medial temporal lobe structures (Chun \& Phelps, 1999; Howard et al., 2006; Packard \& Knowlton, 2002a). The fMRI results of
Menghini et al. (2006) suggest that an implicit learning deficit in dyslexia is associated with a level of activation in higher cerebellar and parietal regions (see also the morphometric results reported by Menghini et al. in this volume). These investigators speculate that automatization is required to achieve reading fluency and that the cerebellum might be important for the development of automaticity. However, it is important to note that the development of automaticity does not necessarily overlap with implicit learning. Automaticity can also arise from repetitive application of explicit, conscious procedures, over and over again, until adequate performance is achieved (Cohen, Dunbar, \& McGlelland, 1990; Cohen, Servan-Schreiber, \& McClelland, 1992; Logan, 1988; MacLeod \& Dunbar, 1988; Petersson, Elfgren, \& Ingvar, 1997, 1999; Petersson, Sandblom, Gisselgård, \& Ingvar, 2001). Howard et al. (2006) also reported significant positive correlations between measures of reading ability and accuracy-based implicit acquisition measures. Notably, they were able to rule out several nonspecific explanations for their results, including a general cognitive or attention deficit, task difficulty, or age, and established that deficits in implicit sequence learning occur even when explicit learning can be ruled out. They emphasize that dyslexics do not suffer from a general implicit learning deficit, but that this deficit is specific to sequential processing, highlighting the importance of sequence complexity (i.e., the level of structure present in the sequences), consistent with the findings of Vicari et al. (2003, 2005).

Much less is known about the implicit acquisition of artificial grammars in dyslexics. To date, only two studies on dyslexia have been conducted using this paradigm. Rüsseler et al. (2006) used a short acquisition session and reported null findings only in terms of correct responses on the grammaticality classification task; no baseline classification was included in the experiment, and they did not control for local substring regularities (i.e., ACS-type information, cf. below). Although there was 
no significant difference between the dyslexic and normal readers, the dyslexic subjects performed at a lower level (mean number of correct responses) on the classification task. In an interesting study by Pothos and Kirk (2004), the artificial grammar of Knowlton and Squire (1996) was used in two AGL tasks of equal formal complexity, but with different stimulus format in a between-subject design: in one of the tasks (the geometric-shapes-embedded AGL task), the stimuli were created so as to encourage whole stimulus perception, deemphasizing the constituent elements, while in the other task (geometric-shapes-sequential AGL task) the constituent elements were emphasized by presenting them serially. Pothos and Kirk (2004) controlled for local substring regularities (i.e., ACS), but did not manipulate this dimension experimentally. The dyslexic group performed equally well on the "grammaticality" classification in both tasks, and the nondyslexic group performed as well as the dyslexic group on the visual-embedded, but less well on the visual-sequential task. These findings were interpreted as indicating that the dyslexic participants were less able to process the individual stimulus elements, suggesting that dyslexic individuals are sometimes prevented from adopting an explicit strategy, which would have interfered with the implicit acquisition mechanisms supporting geometric-sequential AGL. This is consistent with recent work associating dyslexia with problems in focused attention and attention shifting. Thus, Pothos and Kirk (2004) proposed that competent real-world learning is achieved via an interaction of implicit and explicit learning processes.

In summary, there is a cumulating series of investigations of implicit learning in dyslexia, and taken as a whole, these studies suggest that there are aspects of implicit learning that might operate at subnormal levels in dyslexic individuals. The lack of a developmental design in these studies (Goswami, 2003) prevents us from making any conclusions concerning the causal role of an implicit acquisition deficit in dyslexia. It might be an outcome of dyslexia rather than a cause, similar in character to the many parallel findings between the dyslexic and illiterate brain (Petersson et al., 2009; Petersson \& Reis, 2006; Petersson, Reis, et al., 2001). A few tentative conclusions are warranted, however: (a) dyslexia does not seem to be associated with a general implicit learning deficit; (b) the implicit learning deficit observed does not seem to be related to nonspecific factors such as general cognitive or attention deficit, task difficulty, or age; (c) the implicit acquisition deficit seems to be related to sequence processing, which is likely related to sequence complexity (i.e., the level of structure present in the sequences; for a short review see Petersson, 2005b) and for a comprehensive review see Davis et al., 1994); and (d) the implicit learning deficit in dyslexia can be observed when explicit learning is intact.

\section{Implicit Artificial Grammar Acquisition}

The artificial grammar used by Reber (1967), here and subsequently referred to as the Reber grammar, is an example of a right-linear phrase structure grammar which generates a rational language (Perrin \& Pin, 2004). This type of grammar represents the simplest formal model that captures the idea of the "infinite use of finite means" (Petersson, 2005b; Petersson, Grenholm, \& Forkstam, 2005). The Reber grammar, like any right-linear phrase structure grammar, can be implemented in a finite-state architecture (see Fig. 1; from Petersson, 2005). We used this grammar in the present study as a generator of the stimulus material. The finitestate machine can be viewed as an explicit generating mechanism and as a recognition device for a formal language (e.g., Davis et al., 1994). In general, a formal (artificial) grammar serves as an intentional definition (Chomsky, 1986) of a language, and represents a formal specification of the mechanism that generates structural regularities in the output. Here, it should be noted that the term language in formal language is technical and does not entail anything beyond what is outlined above and that a formal (or 


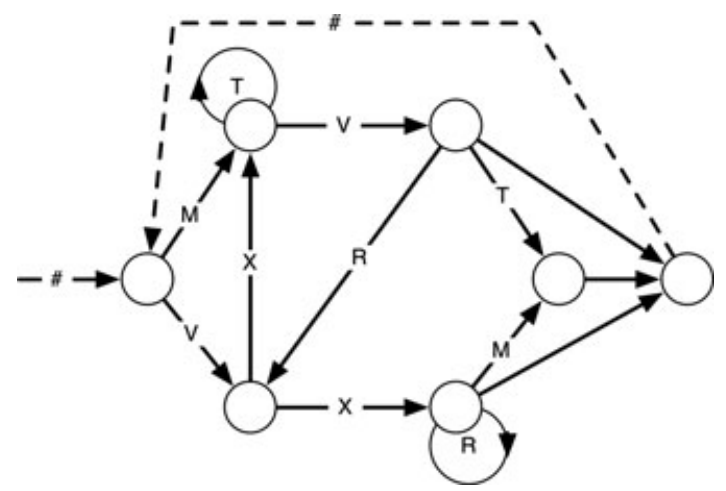

Figure 1. The transition graph representation of the Reber grammar, which was used to generate the grammatical strings in the present study (Petersson, 2005).

artificial) grammar represents a specification of a mechanism that generates (or recognizes) certain types of sequential structural regularities (Petersson et al., 2004). It is also important to note that the finite-state architecture is not limited to capturing local substring dependencies, but that this architecture can also incorporate long-distance dependencies (as long as there is a fixed finite upper bound for these dependencies; cf. Petersson, 2005b; Petersson et al., 2005).

The typical artificial grammar learning (AGL) experiment includes an acquisition phase followed by a grammaticality classification. During the acquisition phase, participants are engaged in a short-term memory task using an acquisition sample of symbol sequences generated from an artificial grammar. Subsequent to the acquisition phase, subjects are informed that the symbol sequences were generated according to a complex system of rules and they are asked to classify new items as grammatical or not based on their immediate impression (guessing based on "gut feeling"). The subjects typically perform reliably above chance (Forkstam et al., 2006; Petersson et al., 2004), and it can be concluded that participants have acquired knowledge about aspects of the underlying generative structure. It is assumed that the classification performance is based on implicit acquisition mechanisms because subjects are typically unable to provide sufficient reasons to motivate their classification decisions (Forkstam et al., 2006; Forkstam \& Petersson, 2005; Stadler \& Frensch, 1998). An alternative way of assessing the implicit acquisition of an artificial grammar is the structural mereexposure version of AGL. This version is based on the "mere exposure" effect, which refers to the finding that repeated exposure to a stimulus induces an increased preference for that stimulus compared to novel stimuli (Zajonc, 1968). The mere-exposure version might be a more sensitive measure of implicitly acquired knowledge because the participants are never made aware of the existence of an underlying generative mechanism.

\section{Goals of the Current Study}

In the present study we characterize a new forced-choice structural mere-exposure AGL paradigm in normal readers based on preference classification. We compare this preference classification paradigm with the standard grammaticality classification paradigm. We predict, on the basis of the mere-exposure effect (Zajonc, 1968), that the development of preference will start to correlate with grammaticality. In order to achieve these objectives, we used a balanced associative chunk strength (ACS) design (Forkstam et al., 2006; Meulemans \& Van der Linden, 1997). In the balanced ACS design, the factors grammaticality status (grammatical/nongrammatical) and ACS (high/low) are independently controlled in a $2 \times 2$ factorial design. It has been argued that sensitivity to the level of ACS is a reflection of a statistical fragment-based learning mechanism, whereas sensitivity to the grammaticality status of the items, independent of ACS, is related to an implicit structure-based acquisition mechanism. Moreover, it is not implausible that learning based on ACS reflects an explicit declarative memory mechanism involving the medial temporal lobe (Forkstam et al., 2006; Lieberman, Chang, Chiao, Bookheimer, \& Knowlton, 2004), while implicit learning 
of grammaticality status independent of ACS reflects a procedural learning mechanism involving the basal ganglia and the prefrontal cortex (Forkstam et al., 2006). In this study, the subjects participated in one implicit acquisition session per day for 5 days; symbol strings were presented visually one letter at a time, which requires temporal integration of information. Before the first acquisition session on the first day, subjects participated in a baseline preference classification task. Finally, after the last acquisition session on the 5th day, subjects performed a preference classification and then the standard grammaticality classification task.

In summary, the objectives of the present study were to investigate the behavioral equivalence of the forced-choice preference classification to the standard grammaticality classification task and to explore the effects of the factors (a) grammaticality status (grammatical/nongrammatical) and (b) ACS (high/low) on the classification task (preference/grammaticality). In the Discussion section, we argue that these AGL paradigms are suitable for further investigation of the implicit learning characteristics in dyslexia.

\section{Methods}

\section{Participants}

Thirty-two right-handed (16 females and 16 males; mean age $\pm \mathrm{SD}=22 \pm 3$ years; mean years of education $\pm \mathrm{SD}=16 \pm 2$ ), healthy Dutch university students volunteered to participate in the study (part of a larger fMRI project; data not shown). They were all prescreened, and none of the subjects used any medication, had a history of drug abuse, head trauma, neurological or psychiatric illness, or a family history of neurological or psychiatric illness. All subjects had normal or corrected-tonormal vision. Written informed consent was obtained from all participants according to the protocol of the Declaration of Helsinki, and the local medical ethics committee approved the study.

\section{Stimulus Material}

We generated 569 grammatical $(G)$ strings from the Reber grammar (5-12 consonants from the alphabet [M, S, V, R, X]; see Fig. 1). For each item we calculated frequency distribution of 2- and 3-letter chunks for both terminal and complete string positions in order to derive the associative chunk strength (ACS) for each item (cf. Knowlton \& Squire, 1996; Meulemans \& Van der Linden, 1997). Then, iteratively, we randomly selected 100 strings, generating an acquisition set that was comparable in terms of 2- and 3-letter chunks to the complete string set. Subsequently we generated the nongrammatical string, derived from each remaining grammatical string by a switch of letters in two nonterminal positions, and these were selected to match the grammatical strings in terms of both terminal and complete string ACS (i.e., collapsed over order information within strings). Finally, in an iterative procedure, we randomly selected three sets of 60 strings each from the remaining grammatical strings, in order to generate the three classification sets consisting of $50 \%$ grammatical and nongrammatical strings, as well as 50\% high and low ACS strings relative to ACS information in the acquisition set and independent of grammaticality status. Thus the stimulus material included an acquisition set and two classification sets (all sets were disjoint). The classification sets were used for the $2 \times 2$ factorial design of the classification task. Thus each classification set consisted of 30 strings of each string type: high ACS grammatical (HG), low ACS grammatical (LG), high ACS nongrammatical (HNG), and low ACS nongrammatical (LNG).

\section{Experimental Procedures}

The subjects were informed on the first day that they were to participate in a shortterm memory experiment. The complete 
experiment was conducted over 5 days with an acquisition session each day. An initial preference classification test (PG) was performed before the first acquisition session on the first day (AGL1). A second preference test was performed after the last session on the 5th day (AGL2). After the AGL2, the grammaticality instruction was introduced (AGL3). During both acquisition and classification sessions, each string was centrally presented letter-by-letter on a computer screen (2.7-6.9 s corresponding to 5-12 letters; $300 \mathrm{~ms}$ letter-presentation duration, $300 \mathrm{~ms}$ interletter interval) using the Presentation software (http://nbs.neuro-bs.com).

\section{Implicit Acquisition Task}

During each acquisition session, all subjects were presented with the 100 acquisition strings (acquisition set) on a computer screen (presentation order randomized for each acquisition session). When the last letter in a string disappeared, the subject was instructed to immediately reconstruct the string from memory by typing on a keyboard in a self-paced fashion. No performance feedback was given and only positive examples (i.e., grammatical strings) were presented during acquisition. The acquisition phase lasted approximately 20-40 min.

\section{Classification Tasks}

The classification task consisted of a yes/no forced-choice procedure, and the subjects were instructed to make their choice based on their immediate impression ("gut feeling"). On the first day, the subjects were given the preference classification instruction (AGL1); they were instructed to classify novel strings as preferable or not (likeable/pleasant or not) and told that there was no right or wrong response. The subjects were given the same preference instruction on the last day (AGL2). After the AGL2, participants were informed about the grammatical nature of the grammar and were instructed to classify new strings as grammatical or not (AGL3). During classification on days 1 and 5, the participants were presented with novel let- ter strings from the classification set in the same way as during acquisition. During a classification session 30 strings were presented one at a time on a computer screen. After a 1-s prestimulus period, the strings were presented for $3 \mathrm{~s}$, followed by a 1-s motor preparation delay period. The subject then had $2.5 \mathrm{~s}$ to make his or her classification decision and push the corresponding key with the left or right index finger, based on the preference. The classification sets and string presentation order were balanced over subjects. Each classification session was split in two in order to balance response finger within subject, each lasting approximately $20 \mathrm{~min}$. The stimuli were presented via an LCD projector, projecting the computer display onto a semitransparent screen that the subject comfortably viewed through a mirror device. At the end of the experimental procedure on day 5, participants were presented with a generation task and then a 31-item fragment-completion task. In the generation task, participants were instructed to generate 10 letter strings that they regarded as grammatical; in the fragmentcompletion task they were instructed to complete each item with the letter they thought would render the string grammatical.

\section{Data Analysis}

Repeated-measures ANOVAs were used for the analysis of the data, unless otherwise stated (statistical software package SPSS). A significance level of $P<0.05$ was used. Scores were based on hit and endorsement rates. The hit rate is defined as the sum of all hits (accepted grammatical strings) and correct rejections (rejected nongrammatical strings). The endorsement rate is defined as the number of all strings classified as grammatical, independent of the actual grammaticality status (cf. Forkstam et al., 2006; Meulemans \& Van der Linden, 1997).

\section{Results}

On the baseline preference classification (AGLl; that is, before any exposure to the 
TABLE 1. Endorsement Rates over Grammaticality and ACS levels ${ }^{a}$

\begin{tabular}{|c|c|c|c|c|c|c|}
\hline & \multicolumn{2}{|c|}{ AGL1 } & \multicolumn{2}{|c|}{ AGL2 } & \multicolumn{2}{|c|}{ AGL3 } \\
\hline & High ACS & Low ACS & High ACS & Low ACS & High ACS & Low ACS \\
\hline G & $53(15) \%$ & $45(18) \%$ & $73(16) \%$ & $62(20) \%$ & $82(20) \%$ & $71(21) \%$ \\
\hline $\mathrm{NG}$ & $51(21) \%$ & $48(13) \%$ & $41(22) \%$ & $34(17) \%$ & $32(22) \%$ & $27(21) \%$ \\
\hline
\end{tabular}

${ }^{a}$ Percentage of items endorsed (i.e., item classified as grammatical independent of actual grammaticality status) by condition (grammatical/nongrammatical $\times$ high/low associative chunk strength (ACS) status; mean performance level and standard deviation). $\mathrm{G}=$ grammatical; $\mathrm{NG}=$ nongrammatical; ACS = associative chunk strength.

grammar) subjects classified at the expected chance level $[50 \pm 7 \%$ correct, $T(31)=0.42$, $P=0.67]$. Consistent with previous findings (Forkstam et al., 2006; Petersson et al., 2004) the overall correct classification performance was clearly above chance on preference [AGL2; $65 \pm 14 \%$ correct, $T(31)=$ 5.7, $P<0.0001]$ and grammaticality classification [AGL3; $73 \pm 16 \%$ correct, $T(31)=$ $7.7, P<0.0001]$. Thus, subjects classified items reliably above chance on both the preference classification (AGL2) and the grammaticality classification (AGL3) tasks. The classification performance improved after the grammaticality instruction was provided $[F(1,31)=8.8$, $P=0.006]$.

\section{Classification Performance: Hit Rates}

The analysis of hit rate (classification performance) showed that the subjects were sensitive to the grammaticality status of the items $[F(2,62)=26, P<0.0001]$. In particular, the participants classified the grammatical strings correctly more often (AGL2 > AGL1), and the hit increased further on the AGL3 task compared to both AGL1 and AGL2. Specific contrast comparisons revealed that the group improved its classification performance for LG strings $[F(2,62)=15.6, P<0.0001]$ and $\mathrm{LNG}$ strings $[F(2,62)=17.8, P<0.0001]$ after the grammaticality instruction (AGL3) in comparison to both the AGL2 and AGL1 tasks, whereas the classification performance for HNG strings improved in comparison only to the AGLl task $[F(2,62)=10.8, P<0.0001]$.

\section{Classification Performance: Endorsement Rates}

We then analyzed the performance data in terms of endorsement rate (i.e., item classified as grammatical independent of actual grammaticality status). Both grammaticality and ACS status influenced the endorsement rate (Table 1, Figs. 2 and 3). A repeated-measures ANOVA with task (AGL1/2/3), grammaticality $(\mathrm{G} / \mathrm{NG})$, and ACS (H/L) as within-factors showed significant main effects of grammaticality $[F(1,31)=47, P<0.0001]$ and ACS $[F(1$, $31)=18, P<0.0001]$, whereas the main effect of task type was nonsignificant $[F(2,62)=1.4$, $P=0.25]$. In addition, there was a significant interaction between task and grammaticality $[F(2,62)=37, P<0.0001]$, while the interaction between task and ACS was nonsignificant $[F(2,62)=0.48, P=0.57]$. This shows that grammaticality is the main contributor to the increased classification performance between the baseline and the two classification tasks after implicit acquisition of the grammar. These results suggest that subjects implicitly acquired knowledge about the underlying grammar after only 5 days of acquisition. Moreover, there was a significant interaction between grammaticality and ACS $[F(1,31)=8.9, P<0.05]$. Post-hoc analysis revealed that this interaction was due to the overall difference in classification performance of AGL2/3 compared to the baseline performance (AGL1), as well as the comparison between AGL2 and AGL3. No other interactions reached significance $(P>0.9)$. 


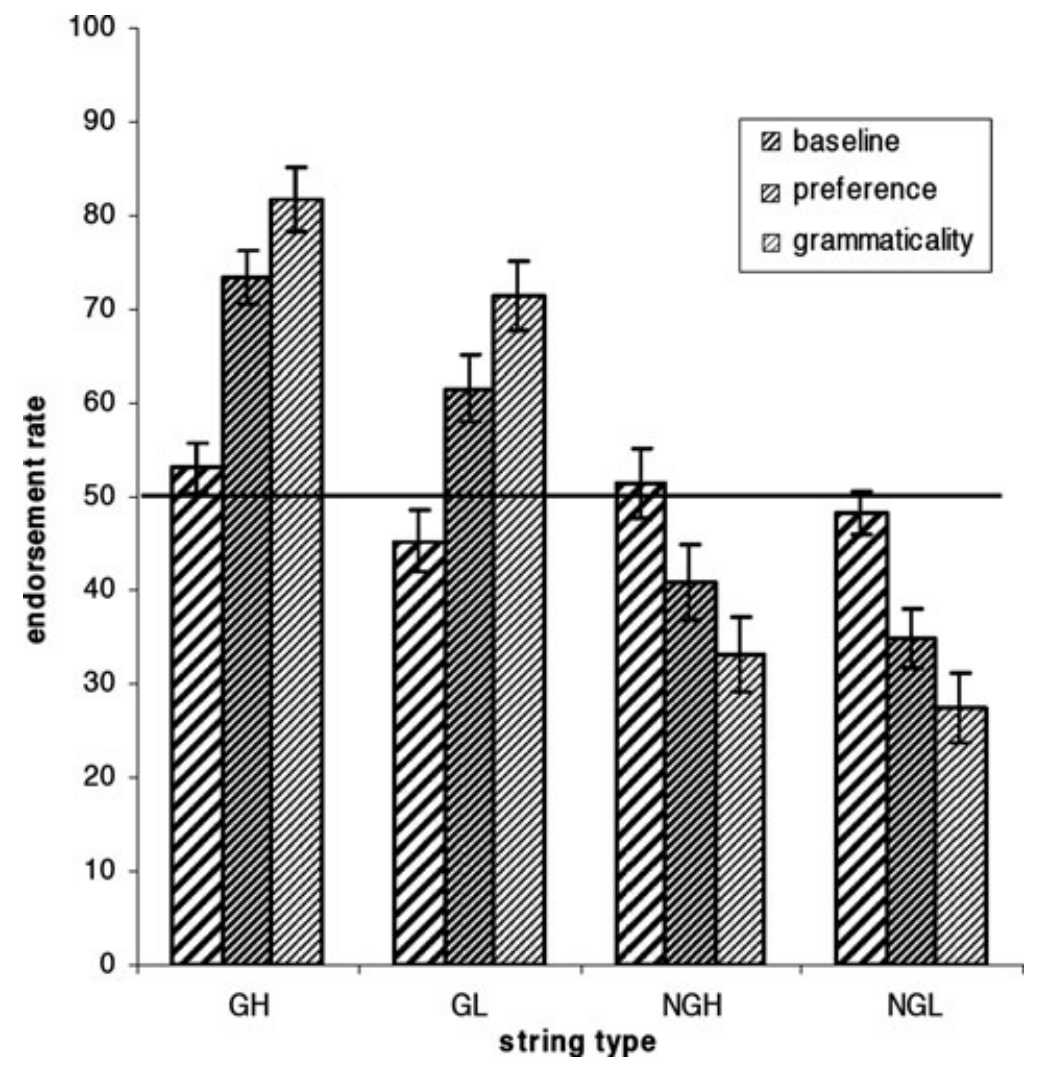

Figure 2. Endorsement rates over grammaticality and ACS levels. The endorsement rates (i.e., item classified as grammatical independent of actual grammaticality status) as a function of grammaticality status as well as associative chunk strength $(\mathrm{GH}$ : grammatical high ACS strings, GL: grammatical low ACS strings, NGH: nongrammatical high ACS strings, NGL: nongrammatical low ACS strings). The endorsement rate of grammatical versus nongrammatical items increases as a function of repeated acquisition for both high and low ACS strings. Error bars correspond to standard error of the mean.

Specifically, preference classification (AGL2) was significantly affected by grammaticality status $[F(1,31)=31.7, P<0.0001]$ and ACS status $[F(1,31)=15.4, P<0.0001]$. These effects were also observed in grammaticality classification [AGL3; grammaticality status, $F(1$, $31)=61.6, P<0.0001$; ACS status, $F(1,31)=$ 13.6, $P<0.001$ ], while the interaction between grammaticality and ACS was not significant on either task [AGL2: $F(1,31)=3.8, P=0.059$; AGL3: $F(1,31)=2.6, P=0.11]$.

We further investigated the effects of grammaticality, following Chang and Knowlton (2004), who argued that ACS might not be a useful cue for the low ACS items, but that correct performance on these items has to be based on knowledge of structural regularities rather than local substring familiarity. Similarly to Lieberman et al. (2004), we found no effects of grammaticality status for both high and low ACS strings on the baseline test [AGL1; HG vs. $\mathrm{HNG}: F(1,31)=0.42, P=0.52 ; \mathrm{LG}$ vs. LNG: $F(1,31)=0.65, P=0.43$ ], while we observed significant effects for preference classification [AGL2; HG vs. HNG: $F(1,31)=$ 34, $P<0.0001$; LG vs. LNG: $F(1,31)=24$, $P<0.0001]$ and grammaticality classification [AGL3; HG vs. HNG: $F(1,31)=59, P<$ 0.0001; LG vs. LNG: $F(1,31)=55, P<$ $0.0001]$. We also observed a significant effect of ACS for both grammatical and nongrammatical strings during preference classification 


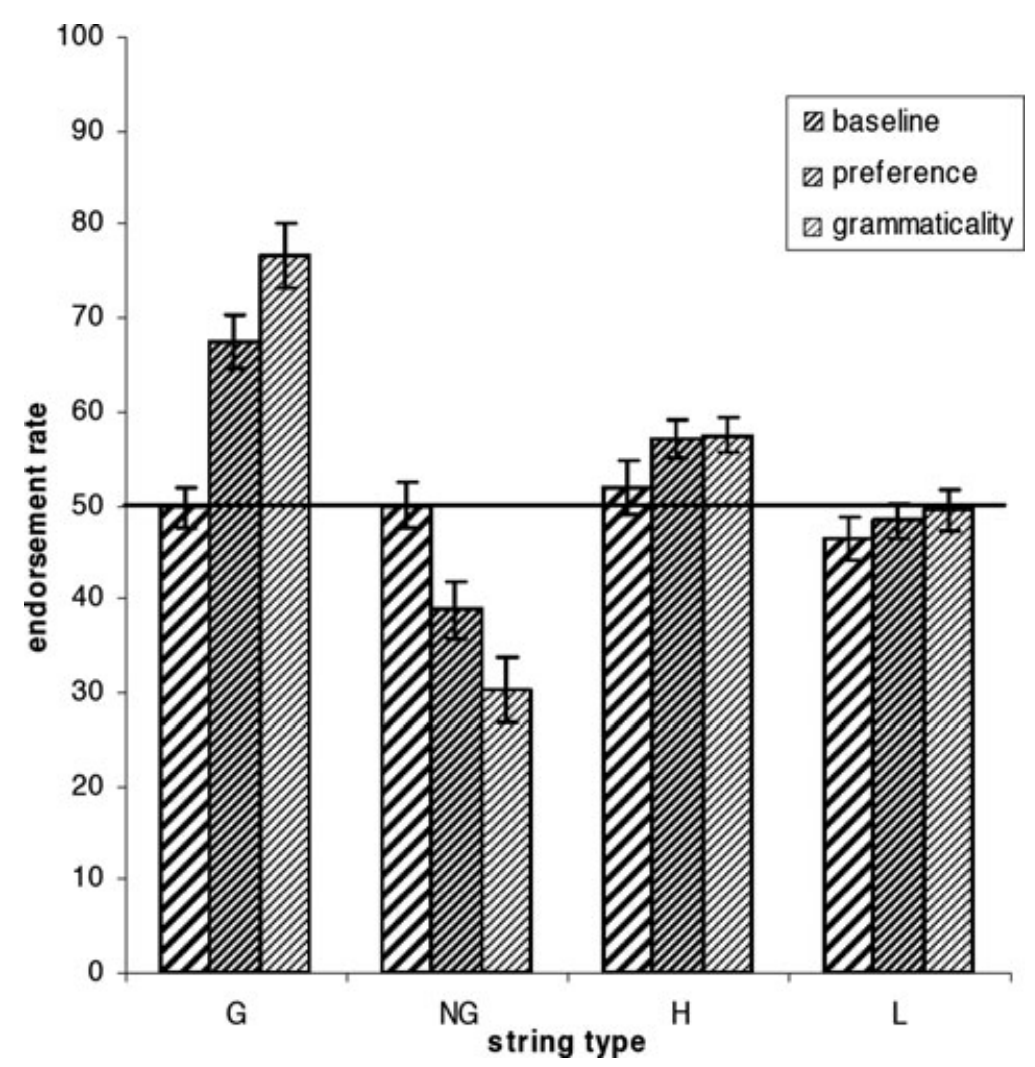

Figure 3. Endorsement rates over grammaticality and ACS main factor categories. The endorsement rates (i.e., item classified as grammatical independent of actual grammaticality status) as a function of grammaticality status ( $G=$ grammatical strings, $N G=$ nongrammatical strings) as well as associative chunk strength $(H=$ high $A C S$ strings, $L=$ low ACS strings). The endorsement rate for grammatical versus nongrammatical items, but not for high versus low ACS items, increases as a function of repeated acquisition sessions. Error bars correspond to standard error of the mean.

[AGL2; HG vs. LG: $F(1,31)=22, P<$ 0.0001; HNG vs. LNG: $F(1,31)=4.04, P=$ $0.05]$. However, for grammaticality classification, ACS only had an effect on the grammatical strings [AGL3; HG vs. LG: $F(1,31)=21$, $P<0.0001]$, but not on the nongrammatical strings (HNG vs. LNG: $F(1,31)=2.9, P=$ 0.09].

In addition, we compared LG versus HNG based on the argument that this maximally contrasts structural versus substring knowledge; if grammaticality status is used for classification, the acceptance of an LG item would crucially depend on the grammaticality status of the item, whereas if substring knowledge is used, the low ACS status would pro- mote a rejection decision. On the other hand, if substring knowledge is used for classification, the acceptance of HNG items would depend on the high ACS status, while if grammatical status is used, the grammaticality status would indicate a rejection decision. As predicted, we found a significant advantage for LG over HNG strings in both preference classification [AGL2; LG > HNG: $T(31)=3.28$, $P=0.003]$ and grammaticality classification [AGL3; LG > HNG; $T(31)=5.82, P<$ $0.0001]$. Taken together, these results show that grammaticality status independent of ACS is used for structural generalization in classifying novel strings and provide support for the notion that grammatical structure other than substring 
or fragment features is used for successful classification.

\section{Signal Detection and Bias: Analysis of the d-Prime and Beta Values}

The subjects showed a stable d-prime effect in discriminating between grammatical $(\mathrm{G})$ and nongrammatical (NG) strings [except in the baseline AGL1 test; mean d-prime values: AGL1 $=0.006$, AGL2 $=0.94$; AGL3 $=$ 1.53; AGL2 > AGL1: $T(31)=-4.91, P=$ 0.0001; AGL3 > AGL2: $T(31)=-2.95$, $P=0.006$, AGL3 > AGL1: $T(31)=-7.63$, $P=0.0001]$. No significant response bias was found (mean beta-values: AGL1 = 1.02; AGL2 $=1.01$; AGL3 $=1.17$; all $P>0.6$ ). However, participants showed no d-prime effect in discriminating between high and low ACS strings (mean d-prime values: AGL1 = 0.15 , AGL2 $=0.22 ;$ AGL3 $=0.21$; all $P>$ 0.66). In other words, no difference in the ability to discriminate better high than low chunks was found. In addition, no significant response bias was observed (mean beta-values: AGL1 = 0.99; AGL2 $=0.98 ;$ AGL3 $=1.00$; all $P>$ $0.8)$. We also investigated the behavioral data for training effects on day 5 by dividing each classification task (preference/grammaticality) into four separate blocks for each task. The statistical analysis yielded no differences in the performance between the four blocks within each classification task.

\section{Subjective Reports}

During each classification session (AGL1/2/3), each subject was asked to rate his or her level of attention, distraction, engagement, boredom, and perceived difficulty ("VAS" ratings four times evenly distributed over each session). There was no significant difference on these measures except for a small increase in the level of attention [AGL1: $7.9 \pm$ 1.1; AGL2: $7.9 \pm 1.1$; AGL3: $8.3 \pm 1.0 ; F(2$, 58) $=9.2, P=0.0001]$ and the participants also rated grammaticality classification as more difficult than preference classification [AGL1: $3.4 \pm$ 2.3; AGL2: $3.2 \pm 2.3$; AGL3: $4.8 \pm 2.8 ; F(2,62)=8.96, P=0.001]$. Most participants reported that the stimuli presented in the classification tasks were similar to what they saw during the acquisition sessions, and they noticed some regularity in the stimulus during the acquisition task (typically, that the strings would start with $\mathrm{M}$ or $\mathrm{V}$ ). All but one participant reported only vague criteria for his or her preference and grammaticality decisions (which would apply equally to all item types).

\section{Fragment Completion and Generation Performance}

A fragment completion and a generation task were administered after the last grammaticality classification AGL3. In the fragment completion task, the participants had to fill in a missing letter in 31 strings that they had never encountered before. All participants scored significantly above chance levels (20\%; mean correct completions $=74 \pm 16 \%$ ). Statistically significant correlation between the number of correct completions and the percentage of correct responses on both classification tasks (AGL2/3) was found (AGL2: $P=0.025$; AGL3: $P=$ $0.013)$. In the generation task, the participants were asked to generate ten grammatically correct strings; 27 participants could generate grammatically correct strings (mean $=$ $5.3 \pm 3.7)$. The generated strings were categorized as new (correct grammatical strings that were never presented to the participants), exact copies (correct grammatical strings that were not new, but already presented during the acquisition sessions), and copies with more or less repeated trigrams. In the latter case, correct grammatical strings were not "new," because if a repeated substring was deleted then they would also have been presented already. For example, if a participant were to write MSVRXVRXVRXVS, and if some but not all of the VRXs are deleted, then the string was part of stimuli of the acquisition sessions. According to this classification, participants 
generated on average 2.3 new grammatical strings and 2.3 exact copies. A significant correlation was found between the generation of grammatical items and the percentage of correct responses on AGL3 $(P=0.0001)$, but not on AGL2. The rating of perceived performance did not correlate with the percentage of correct responses on AGL3 (participants were asked to rate this only after AGL3).

\section{Discussion}

The artificial grammar learning paradigm has been used as a model of several aspects of language acquisition and implicit learning. In this experiment we modified the original version of the paradigm by investigating both grammaticality and preference classification. It can be argued that preference classification might be more optimal in characterizing the outcome of implicit acquisition since in the preference version participants are kept completely unaware of the underlying generative mechanism, whereas in the grammaticality version, the subjects have at least, in principle, been informed about the existence of an underlying complex set of rules at classification (but not during acquisition). The results of the present study showed that the participants implicitly acquired knowledge about the underlying artificial grammar, since participants performed well above chance levels on both preference (AGL2) and grammaticality classification (AGL3) in comparison to baseline classification (AGL1). Participants improved their performance in AGL3 compared to AGL2. Thus the instruction type did influence the final classification proficiency. However, this difference is quantitative rather than qualitative in nature since all effects significant in grammaticality classification (AGL3) were already significant in preference classification (AGL2), as well as the reverse; only the pattern of results was strengthened in AGL3 compared to AGL2. We found that being informed about the existence of an underlying generative mechanism during the utilization of ACS did not increase in grammaticality classification and the significant effect of $L G$ versus $H N G$ was already present in preference classification (AGL2; LG > HNG). These results suggest that grammaticality status independent of ACS is used for structural generalization in classifying novel strings and provides support for the notion that grammatical structure other than local substring regularities is to a large extent used for classification. Thus, the abstraction of grammatical structure takes place during implicit artificial grammar acquisition. This can be seen especially in the quantitative performances between the two classification tasks that take place without an increase in the use of ACS-type information. Furthermore, the use of a preference classification baseline ensures that the effects observed in the classification task are actually attributable to information learned during the acquisition phase. Subjective reports also suggested that the participants did not utilize an explicit rulesearching strategy, but that their classification decisions were reached by guessing based on "gut feeling." In addition, the subjective ratings of perceived performance did not correlate with the actual classification performance. These results show that preference and grammaticality classification are equivalent in terms of behavioral effects and strongly support the notion that humans can implicitly acquire knowledge about a complex system of interacting rules by mere exposure to the acquisition material that can also be effectively put to use (Reber, 1967). In other words, preference starts to correlate with the grammaticality status of the classification items without any explicit awareness of the underlying generative mechanisms as predicted by the mere exposure effect (Zajonc, 1968).

\section{Dual Mechanisms in Implicit Artificial Grammar Acquisition}

Additional support for the implicit character of AGL comes from lesion studies on amnesic patients. Knowlton and Squire (1996) investigated amnesic patients and normal controls 
on the original AGL task as well as a transfer version of the task. The patients and their normal controls performed similarly on both AGL versions, while the amnesic patients could not explicitly retrieve complete strings or any substring information. Knowlton and Squire (1996) argued that AGL depends on implicit acquisition of both abstract (i.e., rule-based) and exemplar-specific information, the latter indicated by the acquisition of distributional information of local substring regularities (i.e., ACS-type information). The acquisition of long-distance dependencies, as opposed to local substring dependencies, has been demonstrated both in visuomotor sequence learning and in AGL (Poletiek, 2002). Moreover, it is known that infants can rapidly acquire and generalize over local sequential regularities, and several studies have shown rapid (on the order of 2-10 min) "rule abstraction" (Marcus, Vijayan, Bandi Rao, \& Vishton, 1999), AGL (Gomez \& Gerken, 1999), and transition probability acquisition in artificial syllable sequences (Saffran, Aslin, \& Newport, 1996) capacities in 8-month-old infants. It is also clear from these studies that distributional information of local sequential regularities are acquired and used for grammaticality classification in addition to implicit abstraction of grammatical structure (Forkstam et al., 2006; Meulemans \& Van der Linden, 1997).

\section{Artificial Grammar Learning and Functional Neuroimaging}

A number of functional magnetic resonance imaging (fMRI) studies have investigated implicit (e.g., Forkstam et al., 2006; Lieberman et al., 2004; Petersson et al., 2004; Seger, Prabhakaran, Poldrack, \& Gabrieli, 2000) and explicit learning of material generated from artificial grammars (e.g., Fletcher, Buchel, Josephs, Friston, \& Dolan, 1999; Strange, Henson, Friston, \& Dolan, 2001) and artificial languages (e.g., Opitz \& Friederici, 2003). In the explicit learning studies (e.g., Fletcher et al., 1999; Opitz \& Friederici, 2003; Strange et al., 2001), the experimental task can be characterized as explicit problem solving with performance feedback (Petersson et al., 2004). In this setup, the participants are explicitly instructed to try to extract the underlying rules based on feedback (trial-and-error search). To overcome the explicit nature of the acquisition task in these experiments, we have, as in this study, investigated grammaticality classification task after implicit acquisition without performance feedback in which the participants only are exposed to positive examples (i.e., well-formed strings, Forkstam et al., 2006; Petersson et al., 2004). The latter two studies showed that artificial syntactic violations activated Broca's region (left Brodmann's area (BA) 44/45; Fig. 4). In Forkstam et al. (2006), the activated frontal regions were more extensive and also included right homotopic regions. Importantly, the left inferior frontal region (BA 45) was the only frontal region that was selectively sensitive to grammaticality, but not to the level of associative chunk strength (ACS). This lends support for the suggestion that the left inferior frontal cortex (BA 45) has a specific role in processing structural regularities (Petersson et al., 2004). This is also consistent with recent results showing that the left prefrontal cortex subserves structured sequence processing (Fig. 4; Bookheimer, 2002; Hagoort, 2005).

A recent study by Lieberman et al. (2004) using an AGL paradigm similar to that of Forkstam et al. (2006) also reported that the caudate nucleus was sensitive to grammaticality (Fig. 4). These findings are in line with a difference between the processing mechanisms that retrieve linguistic structures and the procedural processing mechanisms that apply syntactic "rules" (see, e.g., Ullman, 2004). Here the basal ganglia support the procedural aspects of processing. In this context, it is of interest to note that the basal ganglia learning system (Packard \& Knowlton, 2002b) and the medial temporal lobe memory system (Squire \& Zola-Morgan, 1991) might interact in complex ways, competitively (Poldrack et al., 2001) as well as cooperatively (Voermans et al., 2004). 


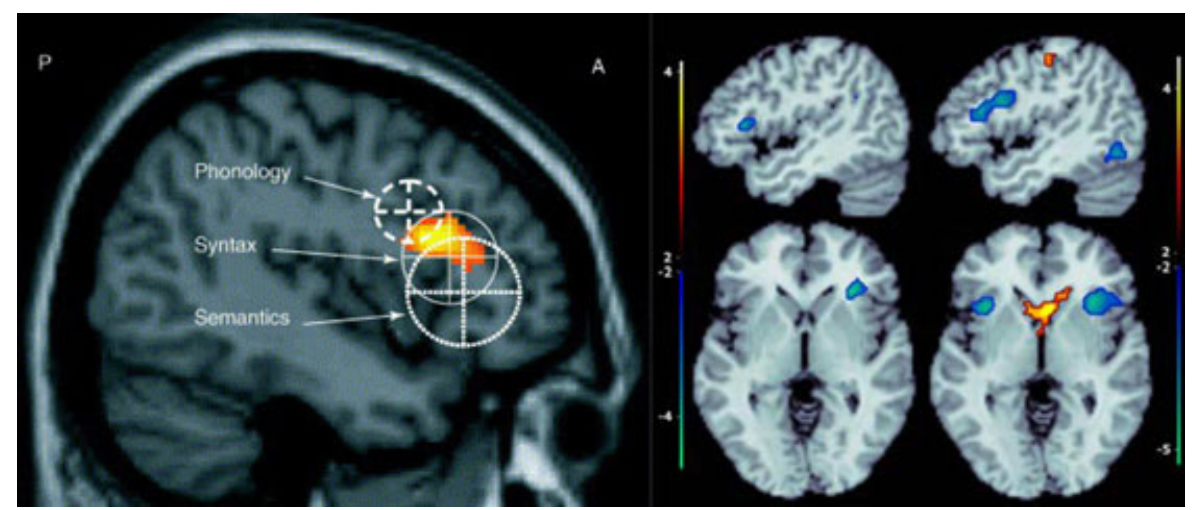

Figure 4. Regions related to phonological, syntactic, and semantic processing (cf. Bookheimer, 2002, and Hagoort, 2005). Left: Activation related to artificial syntactic violations (Petersson et al., 2004). Right: Regions active in artificial grammatical versus nongrammatical items in red and nongrammatical versus grammatical in blue (Forkstam et al., 2006). (In color in Annals online.)

Neural systems supporting procedural learning, and important for online governing of the parsing process, are thought to depend on recurrent networks implemented in corticostriatal loops (see, for example, Luciana, 2003; Nelson \& Webb, 2003). Classifications of G items correlated with the activation of the caudate nucleus when contrasted with NG items. Moreover, the opposite contrast of comparing classifications of $\mathrm{NG}$ versus $\mathrm{G}$ items correlated with activation of the left inferior frontal region. In terms of laterality of the corticostriatal circuits, both the caudate and the inferior frontal region were active bilaterally during processing of grammaticality. The observed selective sensitivity to grammaticality, as opposed to ACS, in the left inferior frontal BA 45 suggests a leftlateral bias in the use of corticostriatal circuits for processing sequence structure.

\section{Artificial Grammar Learning and Dyslexia}

As noted in the introduction, very little is known about the AGL in dyslexic subjects. On the basis of the tentative conclusions outlined in the Introduction, we would like to suggest that the AGL paradigm is a suitable device for further investigation into the implicit learning characteristics in dyslexia. In particular, since the implicit acquisition deficit observed in dyslexia seems to be related to sequence processing and to sequence complexity (i.e., the level of structure present in the sequences), the use of the artificial (formal) language framework for defining and precisely quantifying sequence complexity seems highly relevant (Cutland, 1980; Davis et al., 1994; Hopcroft, Motwani, \& Ullman, 2000). Structural complexity can be systematically varied in artificial grammars, thus making it possible to experimentally manipulate the level of structure available in the stimulus material in a precise and quantitative manner (Petersson, 2005b; Petersson et al., 2005). The AGL paradigm is modality- and material-independent, which allows for experimental investigation into the role of material, sensory modality, and crossmodality transfer effects: for example, results on pure tone- and syllable sequences suggest that performance is higher for syllables compared to pure tone sequences $(P=0.01)$, and while grammaticality classification was significant for both types of sequences on day $5(P<0.001)$, implicit acquisition effects for preference classification on day 5 were only observed for the syllable group $(P=0.01)$ (cf. Faísca, Bramão, Forkstam, Reis, \& Petersson, 2007). As previously illustrated, issues related to temporal-and spatial integration can be investigated as well 
(e.g., Forkstam et al., 2006; Petersson et al., 2004; Pothos \& Kirk, 2004). It is possible to separate the effects related to local substring regularities from those of structure abstraction in a precise manner. Finally, sequence processing after implicit artificial grammar acquisition is known to involve fronto-striatal loops as well as the cerebellum (Forkstam et al., 2006; Lieberman et al., 2004; Petersson et al., 2004), which is relevant given the proposed fronto-striatal-cerebellar circuit deficit in dyslexics (Howard et al., 2006).

Ziegler and Goswami (2005) argue that atypical development of reading skills can arise from variations in the initial conditions or constraints on learning, or from variations in the training environment, or from an interaction between the two. They suggest that explicit access to phonemes is not readily available prior to reading and that all major theories of reading acquisition argue that gaining access to phoneme-size units is a crucial step for the beginning reader of an alphabetic language (Ziegler \& Goswami, 2005). A major cause of the early difficulty of reading acquisition is that phonology and orthography initially favor different grain sizes and that structural regularities present in the lexicon of spoken word forms may form the basis of incidental/implicit learning about phonology (Ziegler and Goswami, 2005). Building on an implicit foundation of phonological knowledge, learning to read involves explicit as well as implicit processes; typically children initially learn grapheme-phoneme mappings explicitly, after which they apply and continue to learn how phonology is mapped onto its written representation implicitly (Gombert, 2003; Petersson \& Reis, 2006; Ziegler \& Goswami, 2005). Ziegler and Goswami (2005) suggest that it is these explicit processes and their potential interactions with the more implicit aspects of lexical processing that are missing from the models. The relationship between reading ability and phoneme awareness is necessarily reciprocal (Petersson et al., 2009; Ziegler \& Goswami, 2005). Awareness of sounds at the smallest grain size (phonemes) does not develop automatically as children get older and the discovery of the phoneme as a psycholinguistic unit depends largely on direct instruction in reading and spelling (Ziegler \& Goswami, 2005). Therefore, in order to fully understand nonoptimal reading and writing development it might be necessary to investigate not only implicit acquisition or explicit learning mechanisms, but their interaction as well.

\section{Conclusions}

In this article we reviewed the literature on implicit learning and dyslexia and tentatively concluded that: (a) dyslexia does not seem to be associated with a general implicit learning deficit; (b) the implicit learning deficit observed in dyslexia does not seem to be related to nonspecific factors like general cognitive or attention deficit, task difficulty, or age; (c) the implicit acquisition deficit seems to be related to sequence processing, which is likely related to sequence complexity; and (d) the implicit learning deficit in dyslexia can be observed when explicit learning is intact. We also characterized a novel forced-choice structural mere-exposure artificial grammar learning paradigm in normal readers in relation to the standard grammaticality classification paradigm. We explored the outcome of an acquisition mechanism capable of extracting structural regularities from experience in an implicit fashion from positive examples alone and without any external supervision or feedback. The results showed that preference and grammaticality classification are equivalent in terms of behavioral effects and strongly support the notion that humans can implicitly acquire knowledge about a complex system of interacting rules by mere exposure to the acquisition material that also can be effectively put to use.

\section{Acknowledgments}

This work was supported by the Max Planck Institute for Psycholinguistics, the F. G. 
Donders Centre for Cognitive Neuroimaging, Vetenskapsrådet (8276), Hedlunds Stiftelse, and Stockholm County Council (ALF, FoUU).

\section{Conflicts of Interest}

The authors declare no conflicts of interest.

\section{References}

Bookheimer, S. 2002. Functional MRI of language: new approaches to understanding the cortical organization of semantic processing. Annual Review of Neuroscience, 25: 151-188.

Chang, G. Y., \& Knowlton, B. J. 2004. Visual feature learning in artificial grammar classification. Fournal of Experimental Psychology: Learning, Memory, and Cognition, 30: 714-722.

Chomsky, N. 1986. Knowledge of language. New York: Praeger.

Chun, M. M., \& Phelps, E. A. 1999. Memory deficits for implicit contextual information in amnesic subjects with hippocampal damage. Nature Neuroscience, 2: 844-847.

Cohen, J. D., Dunbar, K., \& McClelland, J. L. 1990. On the control of automatic processes: a parallel distributed processing model of the Stroop effect. Psychological Review, 99: 45-77.

Cohen, J. D., Servan-Schreiber, D., \& McClelland, J. L. 1992. A parallel distributed processing approach to automaticity. American fournal of Psychology, 105: 239269.

Cutland, N. J. 1980. Computability: An introduction to recursive function theory. Cambridge, UK: Cambridge University Press.

Davis, M. D., Sigal, R., \& Weyuker, E. J. 1994. Computability, complexity, and languages: Fundamentals of theoretical computer science (2nd ed.). San Diego, CA: Academic Press.

Dilling, H., Mombour, W., \& Schmidt, M. H. 1991. International classification of mental diseases: ICD-10. Bern: Huber.

Faísca, L., Bramão, I., Forkstam, C., Reis, A., \& Petersson, K. M. 2007. Implicit learning of structured auditory sequences: An advantage for verbal stimulus. Presented at the Annual Mid-Year Meeting of the International Neuropsychological Society. Bilbao, Spain.

Fletcher, P., Buchel, C., Josephs, O., Friston, K., \& Dolan, R. 1999. Learning-related neuronal responses in prefrontal cortex studied with functional neuroimaging. Cerebral Cortex, 9: 168-178.

Forkstam, C., Hagoort, P., Fernandez, G., Ingvar, M., \& Petersson, K. M. 2006. Neural correlates of artifi- cial syntactic structure classification. NeuroImage, 32: 956-967.

Forkstam, C., \& Petersson, K. M. 2005. Towards an explicit account of implicit learning. Current Opinion in Neurology, 18: 435-441.

Friederici, A. D., Steinhauer, K., \& Pfeifer, E. 2002. Brain signatures of artificial language processing: Evidence challenging the critical period hypothesis. Proeedings of the National Academy of Sciences of the United States of America, 99: 529-534.

Gombert, J. E. 2003. Implicit and explicit learning to read: implication as for subtypes of dyslexia. Current Psychology Letters: Behavior, Brain and Cognition, 10(1):

Gomez, R. L., \& Gerken, L. 1999. Artificial grammar learning by 1-year-olds leads to specific and abstract knowledge. Cognition, 70: 109-135.

Gomez, R. L., \& Gerken, L. 2000. Infant artificial language learning and language acquisition. Trends in Cognitive Sciences, 4: 178-186.

Goswami, U. 2003. Why theories about developmental dyslexia require developmental designs. Trends in $\mathrm{Cog}_{-}$ nitive Sciences, 7: 534-540.

Habib, M. 2000. The neurological basis of developmental dyslexia: an overview and working hypothesis. Brain, 123: 2373-2399.

Hagoort, P. 2005. On Broca, brain and binding: a new framework. Trends in Cognitive Sciences, 9: 416-423.

Hauser, M. D., Chomsky, N., \& Fitch, W. T. 2002. The faculty of language: what is it, who has it, and how did it evolve? Science, 298: $1569-1579$.

Hopcroft, J. E., Motwani, R., \& Ullman, J. D. 2000. Introduction to automata theory, languages, and computation (2nd ed.). Reading, MA: Addison-Wesley.

Howard, J. H., Howard, D. V., Japikse, K. C., \& Eden, G. F. 2006. Dyslexics are impaired on implicit higherorder sequence learning, but not on implicit spatial context learning. Neuropsychologia, 44: 1131-1144.

Karmiloff-Smith, A. 1992. Beyond modularity: a developmental perspective on cognitive science. Cambridge, MA: MIT Press.

Karmiloff-Smith, A., Grant, J., Sims, K., Jones, M. C., \& Cuckle, P. 1996. Rethinking metalinguistic awareness: representing and accessing knowledge about what counts as a word. Cognition, 58: 197-219.

Kelly, S. W., Griffiths, S., \& Frith, U. 2002. Evidence for implicit sequence learning in dyslexia. Dyslexia, 8: 43-52.

Knowlton, B. J., \& Squire, L. R. 1996. Artificial grammar learning depends on implicit acquisition of both abstract and exemplar-specific information. Fournal of Experimental Psychology: Learning, Memory, and Cognition, 22: 169-181.

Lieberman, M. D., Chang, G. Y., Chiao, J., Bookheimer, S. Y., \& Knowlton, B. J. 2004. An event-related fMRI study of artificial grammar learning in a balanced 
chunk strength design. Fournal of Cognitive Neuroscience, 16: $427-438$.

Logan, G. D. 1988. Toward an instance theory of automatization. Psychological Review, 95: 492-527.

Luciana, M. 2003. The neural and functional development of human prefrontal cortex. In M. de Haan \& M. H. Johnson (Eds.), The cognitive neuroscience of development (pp. 157-179). New York: Psychology Press.

MacLeod, C. M., \& Dunbar, K. 1988. Training and Stroop-like interference: evidence for a continuum of automaticity. Fournal of Experimental Psychology: Learning, Memory, and Cognition, 14: 126-135.

Marcus, G. F., Vijayan, S., Bandi Rao, S., \& Vishton, P. M. 1999. Rule learning by seven-month-old infants. Science, 283: 77-80.

Menghini, D., Hagberg, G. E., Caltagirone, C., Petrosini, L., \& Vicari, S. 2006. Implicit learning deficits in dyslexic adults: an fMRI study. NeuroImage, 33: 1218-1226.

Meulemans, T., \& Van Der Linden, M. 1997. Associative chunk strength in artificial grammar learning. fournal of Experimental Psychology: Learning, Memory, and Cognition, 23(4): 1007-1028.

Morais, J. 1993. Phonemic awareness, language and literacy. In R. M. Joshi \& C. K. Leong (Eds.), Reading disabilities: diagnosis and component processes (pp. 175184). Dordrecht, the Netherlands: Kluwer Academic Publishers.

Nelson, C. A., \& Webb, S. J. 2003. A cognitive neuroscience perspective on early memory development. In M. de Haan \& M. H. Johnson (Eds.), The cognitive neuroscience of development (pp. 99-125). New York: Psychology Press.

Opitz, B., \& Friederici, A. D. 2003. Interactions of the hippocampal system and the prefrontal cortex in learning language-like rules. NeuroImage, 19(4): 16301737.

Packard, M. G., \& Knowlton, B. J. 2002a. Learning and memory functions of the basal ganglia. Annual Reviere of Neuroscience, 25: 563-593.

Packard, M. G., \& Knowlton, B. J. 2002b. Learning and memory functions of the basal ganglia. Annual Reviere of Neuroscience, 25: 563-593.

Perrin, D., \& Pin, J.-E. 2004. Infinite words: Automata, semigroups, logic, and games. Amsterdam, the Netherlands: Elsevier.

Petersson, K. M. 2005a. Learning and memory in the human brain. Stockholm: Karolinska University Press.

Petersson, K. M. 2005b. On the relevence of the neurobiological analogue of the finite state architecture. Neurocomputing, 65-66: 825-832.

Petersson, K. M., Elfgren, C., \& Ingvar, M. 1997. A dynamic role of the medial temporal lobe during retrieval of declarative memory in man. NeuroImage, $\mathbf{6 :}$ $1-11$.
Petersson, K. M., Elfgren, G., \& Ingvar, M. 1999. Learning-related effects and functional neuroimaging. Human Brain Mapping, 7(4): 234-243.

Petersson, K. M., Forkstam, C., \& Ingvar, M. 2004. Artificial syntactic violations activate Broca's region. Cognitive Science, 28: 383-407.

Petersson, K. M., Grenholm, P., \& Forkstam, G. 2005. Artificial grammar learning and neural networks. Proceedings of the Cognitive Science Society, 17261731.

Petersson, K. M., Ingvar, M., \& Reis, A. 2009. Language and literacy from a cognitive neuroscience perspective. In D. Olson \& N. Torrance (Eds.), Cambridge handbook of literacy (pp. 152-181). Cambridge, UK: Cambridge University Press.

Petersson, K. M., \& Reis, A. 2006. Characteristics of illiterate and literate cognitive processing: Implications for brain-behavior co-constructivism. In P. B. Baltes \& F. Rösler \& P. A. Reuter-Lorenz (Eds.), Lifespan development and the brain: The perspective of biocultural coconstructivism (pp. 279-305). New York: Cambridge University Press.

Petersson, K. M., Reis, A., Askelöf, S., Castro-Caldas, A., \& Ingvar, M. 2000. Language processing modulated by literacy: a network-analysis of verbal repetition in literate and illiterate subjects. Fournal of Cognitive Neuroscience, 12: 364-382.

Petersson, K. M., Reis, A., \& Ingvar, M. 2001. Cognitive processing in literate and illiterate subjects: a review of some recent behavioral and functional data. Scandinavian Fournal of Psychology, 42: 251-167.

Petersson, K. M., Sandblom, J., Gisselgård, J., \& Ingvar, M. 2001. Learning related modulation of functional retrieval networks in man. Scandinavian fournal of Psychology, 42: 197-216.

Poldrack, R. A., Clark, J., Pare-Blagoev, E. J., Shohamy, D., Creso Moyano, J., Myers, C., et al. 2001. Interactive memory systems in the human brain. Nature, 414: 546-550.

Poletiek, F. H. 2002. Implicit learning of a recursive rule in an artificial grammar. Acta Psychologica, 111: 323335 .

Pothos, E. M., \& Kirk, J. 2004. Investigating learning deficits associated with dyslexia. Dyslexia, 10: $61-$ 76.

Reber, A. S. 1967. Implicit learning of artificial grammars. Fournal of Verbal Learning and Verbal Behavior, 5: 855-863.

Reber, A. S. 1993. Implicit learning and tacit knowledge: Anessay on the cognitive unconscious. New York: Oxford University Press.

Rüsseler,J., Gerth, I., \& Münte, T. F. 2006. Implicit learning is intact in adult developmental dyslexic readers: evidence from the serial reaction time task and artificial grammar learning implicit learning in dyslexia. 
Journal of Clinical and Experimental Neuropsychology, 28: 808-827.

Saffran, J. R., Aslin, R. N., \& Newport, E. L. 1996. Statistical learning by 8-month-old infants. Science, 274: 1926-1928.

Seger, G. A. 1994. Implicit learning. Psychological Bulletin, 115: 163-196.

Seger, C. A., Prabhakaran, V., Poldrack, R. A., \& Gabrieli, J. D. 2000. Neural activity differs between explicit and implicit learning of artificial grammar strings: an fMRI study. Psychobiology, 28: 283-292.

Shaywitz, S. E. 1998. Dyslexia. New England fournal of Medicine, 338: 307-312.

Sperling, A. J., Lu, Z. L., \& Manis, F. R. 2004. Slower implicit categorical learning in adult poor readers. Annals of Dyslexia, 54: 281-303.

Squire, L. R., \& Zola-Morgan, S. 1991. The medial temporal lobe memory system. Science, 253: 1380 1386.

Stadler, M. A., \& Frensch, P. A. (Eds.). 1998. Handbook of implicit learning. Thousand Oaks, CA: Sage.

Stoodley, C. J., Harrison, E. P., \& Stein, J. F. 2006. Implicit motor learning deficits in dyslexic adults. Neurosychologia, 44: 795-798.

Strange, B. A., Henson, R. N. A., Friston, K. J., \& Dolan, R. J. 2001. Anterior prefrontal cortex mediates rule learning in humans. Cerebral Cortex, 11: 10401046 .
Ullman, M. T. 2004. Contributions of memory circuits to language: the declarative/procedural model. Cognition, 92: 231-270.

Vicari, S., Finzi, A., Menghini, D., Marotta, L., Baldi, S., \& Petrosini, L. 2005. Do children with developmental dyslexia have an implicit? Fournal of Neurology, Neurosurgery, and Psychiatry, 76: 1392-1397.

Vicari, S., Marotta, L., Menghini, D., Molinari, M., \& Petrosini, L. 2003. Implicit learning deficit in children with developmental dyslexia. Neuropsychologia, 41: $108-114$.

Voermans, N. C., Petersson, K. M., Daudey, L., Weber, B., van Spaendonck, K. P., Kremer, H. P. H., et al. 2004. Interaction between the human hippocampus and caudate nucleus during route recognition. $\mathrm{Neu}$ ron, 43: 427-435.

Waber, D. P., Marcus, D. J., Forbes, P. W., Bellinger, D. C., Weiler, M. D., Sorensen, L. G., et al. 2003. Motor sequence learning and reading ability: Is poor reading associated with sequencing deficits? Journal of Experimental Child Psychology, 84: 338-354.

Zajonc, R. B. 1968. Attitudinal effects of mere exposure. Fournal of Personality and Social Psychology Monograph Supplement, 9(2): Part 2.

Ziegler, J. C., \& Goswami, U. 2005. Reading acquisition, developmental dyslexia, and skilled reading across languages: a psycholinguistic grain size theory. Psychological Bulletin, 131: 3-29. 